\title{
Ischemic preconditioning reduces peripheral oxidative damage associated with brain ischemia in rats
}

\section{S.S. Frassetto ${ }^{1}$, M.R.C. Schetinger ${ }^{2}$, A. Webber ${ }^{1}$, J.J.F. Sarkis ${ }^{1}$ and C.A. N etto ${ }^{1}$}

\author{
1Departamento de Bioquímica, Instituto de Ciências Básicas da Saúde, \\ Universidade Federal do Rio Grande do Sul, Porto Alegre, RS, Brasil \\ ${ }^{2}$ Setor de Bioquímica, Departamento de Química, Centro de Ciências \\ Naturais e Exatas, Universidade Federal de Santa Maria, Santa Maria, RS, Brasil
}

\section{Correspondence \\ C.A. Netto \\ Departamento de Bioquímica Instituto de Ciências Básicas da Saúde, UFRGS \\ Rua Ramiro Barcelos, 2600 (anexo) \\ 90035-003 Porto Alegre, RS \\ Brasil \\ Fax: + 55-51-316-5535 \\ E-mail: alexneto@ vortex.ufrgs.br \\ Research supported in part by FINEP and CNPq. S.S. Frassetto was the recipient of a CAPES fellowship.}

Received February 2, 1999 Accepted July 26, 1999

\section{Abstract}

Brain ischemia followed by reperfusion causes neuronal death related to oxidative damage. Furthermore, it has been reported that subjects suffering from ischemic cerebrovascular disorders exhibit changes in circulating platelet aggregation, a characteristic that might be important for their clinical outcome. In the present investigation we studied tert-butyl hydroperoxide-initiated plasma chemiluminescence and thiol content as measures of peripheral oxidative damage in naive and preconditioned rats submitted to forebrain ischemia produced by the 4-vessel occlusion method. Rats were submitted to 2 or $10 \mathrm{~min}$ of global transient forebrain ischemia followed by $60 \mathrm{~min}$ or $1,2,5,10$ or 30 days of reperfusion. Preconditioned rats were submitted to a 10min ischemic episode 1 day after a $2-\mathrm{min}$ ischemic event $(2+10 \mathrm{~min})$, followed by $60 \mathrm{~min}$ or 1 or 2 days of reperfusion. It has been demonstrated that such preconditioning protects against neuronal death in rats and gerbils submitted to a lethal $(10 \mathrm{~min})$ ischemic episode. The results show that both 2 and $10 \mathrm{~min}$ of ischemia cause an increase of plasma chemiluminescence when compared to control and sham rats. In the 2-min ischemic group, the effect was not present after reperfusion. In the 10-min ischemic group, the increase was present up to 1 day after recirculation and values returned to control levels after 2 days. However, rats preconditioned to ischemia $(2+10 \mathrm{~min})$ and reperfusion showed no differences in plasma chemiluminescence when compared to controls. We also analyzed plasma thiol content since it has been described that sulfhydryl $(\mathrm{SH})$ groups significantly contribute to the antioxidant capacity of plasma. There was a significant decrease of plasma thiol content after 2, 10 and $2+10$ min of ischemia followed by reperfusion when compared to controls. We conclude that ischemia may cause, along with brain oxidative damage and cell death, a peripheral oxidative damage that is reduced by the preconditioning phenomenon.

\section{Introduction}

Neuronal damage following transient brain ischemia is mediated by various mechanisms, among which reactive oxygen spe-

\section{Key words}

- Brain ischemia

- Ischemic preconditioning

- Oxidative damage

- Hydroperoxide-initiated

chemiluminescence

- Plasma thiols cies-mediated processes play a central role. The injurious effect of free radicals in brain ischemia is evident from studies demonstrating that free radical scavengers confer protection from ischemic damage (1), and trans- 
genic mice overexpressing superoxide dismutase exhibit less edema and smaller infarcts when subjected to ischemia (2). Furthermore, damage to the brain is also observed during reperfusion after ischemia (when the oxygen supply is restored to the brain) and may be attributed primarily to the presence of reactive oxygen species that can induce oxidative stress (3). The sources of reactive oxygen species include: a) products from the arachidonic acid cascade, b) metabolism of xanthine by xanthine oxidase (4), and c) release of excitatory amino acids (5).

Besides the effects on the central nervous system, recent studies have reported that peripheral events also occur after ischemic cerebrovascular disease, such as changes of platelet surface glycoproteins (6), platelet activation and erythrocyte aggregation in the circulation $(7,8)$, and diffusion of nitric oxide (NO) produced by neurons into the bloodstream (9). Although these investigators have shown that ischemic cerebrovascular disease induces peripheral effects, it has not been shown if a probable oxidative damage is related to these effects. Salvemini and Botting (10) have suggested that in ischemic heart disease platelet activation is related to oxidative damage. Thus, superoxide anions $\left(\mathrm{O}_{2}{ }^{--}\right)$enhance platelet activation and aggregation in the circulation, while NO is able to inhibit the response of platelets to superoxide (11).

The results of the present study suggest that brain ischemia may cause, along with brain oxidative damage and cell death, a peripheral oxidative damage that is probably related to important alterations such as microthrombus formation during this disease. However, it has been proposed that brain ischemic preconditioning (when brief periods of ischemia precede longer and injurious ischemic episodes) is responsible for attenuation of neural ischemic injury (12). Thus, our aim was also to establish a relationship between ischemic preconditioning and a possible protection against the state of peripheral oxidative damage. To approach this question, we first measured the tertbutyl hydroperoxide-initiated chemiluminescence emission in plasma of naive and preconditioned rats submitted to forebrain ischemia and reperfusion. This assay is based on the determination of stable products or by-products of the reaction chain of lipid peroxidation, a process that follows the production of oxygen free radicals in biological systems, and has been applied to detect the existence of oxidative damage associated with experimental pathological situations (13). We next examined the effect of brain ischemia and preconditioning on plasma thiols because extracellular sulfhydryl $(\mathrm{SH})$ groups have been proposed to serve as scavenging antioxidants (14) and are generally highly effective in reducing ischemia-reperfusion injury (15). Our hypothesis was that brain ischemia leads to a peripheral oxidative damage and that ischemic preconditioning protects against this effect.

\section{Material and Methods}

Tert-butyl hydroperoxide was obtained from Aldrich Chemicals Co. (Milwaukee, WI, USA) and 5,5-dithiobis-(2-nitrobenzoic acid) (DTNB) was obtained from Sigma Chemical Co. (St. Louis, MO, USA). All other reagents were of analytical grade.

\section{Brain ischemia and preconditioning}

Male Wistar rats from our breeding stock, weighing 180-200 g, were maintained on a 12-h light/12-h dark cycle in a constant temperature room. Global transient forebrain ischemia was produced by the four-vessel occlusion method (4-VO) $(16,17)$. Briefly, the vertebral arteries were electrocoagulated through the alar foramina on the first cervical vertebra under halothane anesthesia, and silastic ties were inserted around the carotids and brought to the surface. Twenty-four hours 
later the ties were tightly clamped for 2 or 10 min (single-ischemic rats). Variable periods of reperfusion were then allowed, i.e., 60 $\min$ or 1,2, 5, 10 and 30 days. The preconditioned group (double-ischemic rats) received 10 -min ischemia 1 day after a 2-min ischemic episode (referred to as $2^{\prime}+10^{\prime}$ ), followed by $60 \mathrm{~min}$ or 1 and 2 days of reperfusion. Rats that did not lose the righting reflex or that convulsed during the ischemia or reperfusion period were discarded. Shamoperated and intact rats were used as controls. Animals were killed by decapitation and blood was collected into citrated tubes. All animal use procedures were approved by the local Animal Care Committee.

\section{Plasma isolation}

Plasma was obtained from fresh citrated rat blood. Red blood cells and buffy coat were removed by centrifugation at $200 \mathrm{~g}$ for $5 \mathrm{~min}$ at room temperature and platelets were removed by plasma centrifugation at $200 \mathrm{~g}$ for $20 \mathrm{~min}$. The supernatant was used for subsequent experiments.

\section{Determination of plasma tert-butyl hydroperoxide-initiated chemiluminescence}

Chemiluminescence emission was measured with an LKB Rack Beta liquid scintillation spectrometer model 1215 (LKBProdukter AB, Bromma, Sweden). Plasma samples in glass vials were kept in the dark up to the moment of assay and determinations were carried out in a dark room in order to avoid vial phosphorescence activated by fluorescent light. When tert-butyl hydroperoxide was added to plasma the emission increased with time to reach a maximal level after $30 \mathrm{~min}$. Thus, we analyzed chemiluminescence emissions $30 \mathrm{~min}$ after the addition of tert-butyl hydroperoxide. Assay conditions as described by Flecha and colleagues (18) were: 1 to $1.5 \mathrm{mg} / \mathrm{ml}$ of plasma protein in a reaction medium consisting of $120 \mathrm{mM}$
$\mathrm{KCl}, 30 \mathrm{mM}$ phosphate buffer, $\mathrm{pH}$ 7.4, plus $3 \mathrm{mM}$ tert-butyl hydroperoxide, in a final volume of $3 \mathrm{ml}$. Results are reported as counts per second (cps)/mg protein.

\section{D etermination of plasma thiol groups}

Plasma SH groups (which originate predominantly from plasma proteins) were measured spectrophotometrically after reaction with DTNB (19). Absorbance at $412 \mathrm{~nm}$ was measured after 20-min incubation at room temperature. Plasma SH groups were calculated using an absorptivity of $13600 \mathrm{~cm} / \mathrm{M}$.

\section{Protein determination}

Protein was determined by the Coomassie blue method (20) with bovine serum albumin used as standard.

\section{Statistical analysis}

Data were analyzed by one-way and twoway analysis of variance. Post hoc tests included the Duncan procedure for multiple intergroup comparisons. $P$ values less than 0.05 were considered to be significant.

\section{Results and Discussion}

\section{Effects of single ischemic episodes and preconditioning to brain ischemia on plasma tert-butyl hydroperoxide-initiated chemiluminescence and plasma thiols}

Plasma tert-butyl hydroperoxide-initiated chemiluminescence emission was determined in rats submitted to 2- and 10-min brain ischemia. We also studied the effect of preconditioning to brain ischemia (doubleischemic episode $=2^{\prime}+10^{\prime}$ ), i.e., a brief ischemic episode $(2 \mathrm{~min})$ inflicted 1 day before a longer period of ischemia (10 min). Several studies have suggested that the ischemic injury is markedly limited when the preconditioning phenomenon occurs, be- 
cause the brief episode of ischemia that causes no neuronal death induces tolerance to the longer ischemic episode (21). Plasma hydroperoxide-initiated chemiluminescence of rats submitted to 2- and 10-min brain ischemia was significantly increased by about $40-50 \%$ when compared to the photoemission of plasma from control rats (Figures $1 \mathrm{~A}$ and 2A). In contrast to 2- or 10-min ischemic episodes alone, plasma from preconditioned rats (submitted to the double-ischemic episode) did not show an increase in emission when compared to control groups or to the
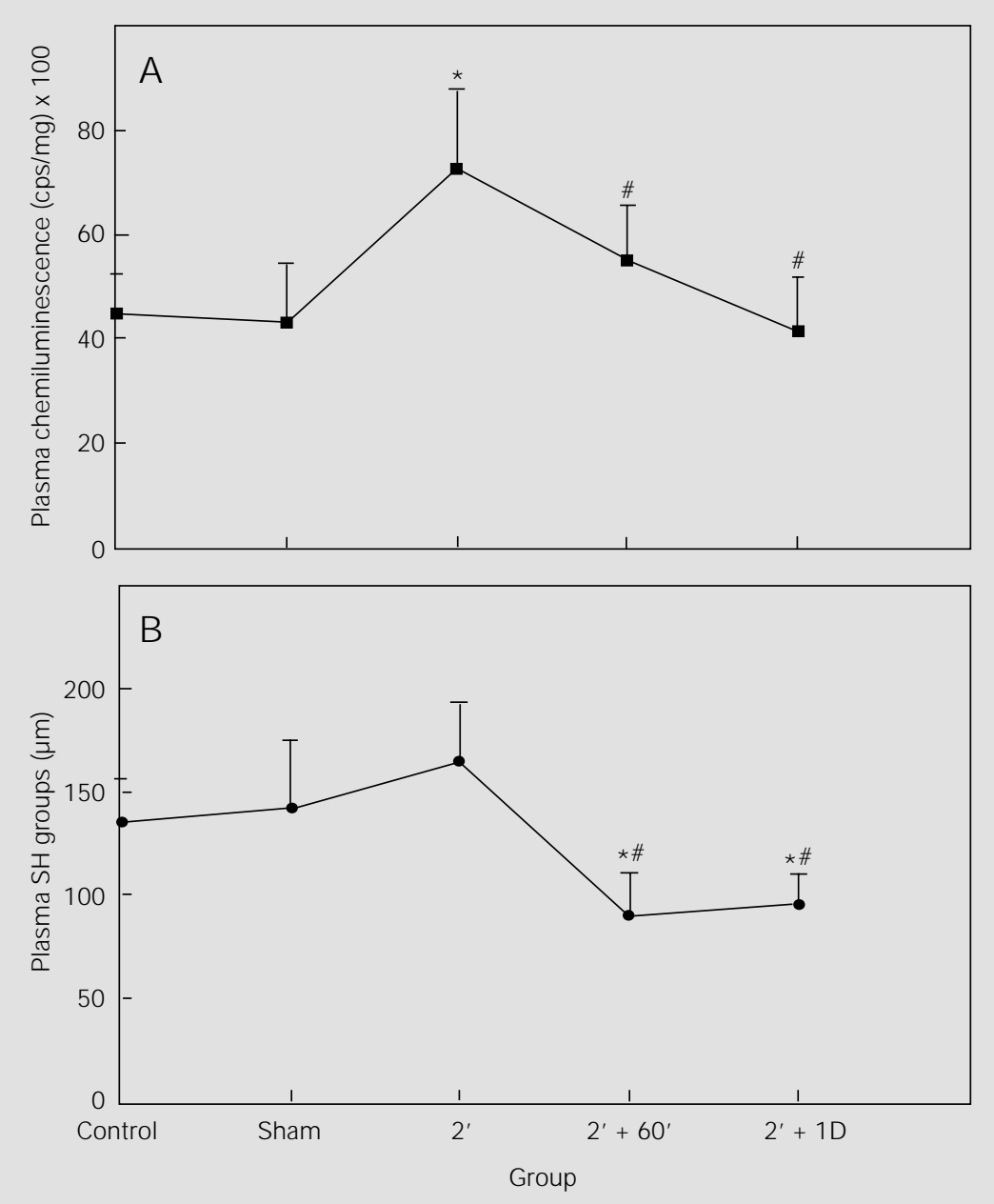

Figure 1 - Effect of 2-min (2') transient forebrain ischemia and reperfusion in rats on tertbutyl hydroperoxide-initiated chemiluminescence of plasma (A) and plasma thiols (B). Rats were submitted to 2 -min (2') ischemia followed by 60 min or 1 day (D) of reperfusion. Data represent the mean $\pm \mathrm{SD}$ for six to nine different animals. $* \mathrm{P}<0.05$ compared to control groups. ${ }^{\mathrm{P}}<0.05$ compared to the 2 -min ( $\left.2^{\prime}\right)$ ischemic group (Duncan multiple range test). $\mathrm{SH}$, Sulfhydryl. group that was submitted to only 2 -min ischemia followed by 1-day reperfusion (Figure $3 \mathrm{~A})$. The fact that single ischemic episodes ( 2 or $10 \mathrm{~min}$ ) show an increase in plasma chemiluminescence emission implies that brain ischemia may induce peripheral oxidative damage. Since brain ischemia increased plasma hydroperoxide-initiated chemiluminescence, we next examined its effects on plasma $\mathrm{SH}$ concentration. The thiols in plasma make a significant contribution to the peroxyl-radical scavenging capacity of plasma (21). Brain ischemia (2 or $10 \mathrm{~min}$ ) and the double-ischemic episode ( $2^{\prime}$ $+10^{\prime}$ ) did not cause alteration of plasma $\mathrm{SH}$ groups (Figures 1B, 2B and 3B).

\section{Effects of reperfusion after single ischemic episodes and preconditioning on plasma tert-butyl hydroperoxide-initiated chemiluminescence and plasma thiols}

Figure 1A illustrates the effect of 60-min and 1-day reperfusion after 2-min brain ischemia on plasma chemiluminescence. As can be seen, reperfusion significantly decreased chemiluminescence emission to control levels when these data were compared to 2-min ischemia. Compared to the effect of reperfusion after 2-min ischemia on plasma thiols (Figure 1B), 60-min and 1-day reperfusion resulted in a significant decrease in SH groups to levels below the control and 2min ischemia values. On the other hand, 60min and 1-day reperfusion after 10-min brain ischemia still resulted in a $30-40 \%$ increase in plasma chemiluminescence emission (Figure 2A). Values for plasma chemiluminescence of rats submitted to 2-, 5-, 10- and 30day reperfusion after 10-min ischemia were similar to controls (Figure 2A). Similar to the results of reperfusion after 2-min ischemia, reperfusion (60 $\mathrm{min}, 1,2,5$ and 10 days) after 10-min ischemia also reduced plasma SH when compared to data from control and 10-min ischemia without reperfusion (Figure 2B). Although the effect of 
reperfusion after 10-min ischemia on the reduction of plasma thiols was observed up to 10 days, values for plasma $\mathrm{SH}$ groups after 30-day reperfusion were not significantly different from controls (Figure 2B), probably because protein thiols (albumin) have a half-life of 20 days (14).

A possible interpretation of these results is that reperfusion after brain ischemia may also induce peripheral alterations in plasma. The results from animals reperfused after 10 -min ischemia indicate that there is a relationship between plasma oxidative damage and brain reperfusion when this injurious ischemic episode occurs, since 60-min and 1-day reperfusion still maintain the increase in chemiluminescence emission caused by 10-min ischemia even when plasma thiols are decreased (Figure 2A). In contrast, reperfusion after a brief ischemic episode (2 min) may lead to the utilization of plasma thiols as antioxidants and, consequently, to a decrease of chemiluminescence emission to control values (Figure 1A). Thus, it seems that the decrease of plasma chemiluminescence emission to control levels during longer periods of reperfusion after 10-min ischemia (Figure 2A) may also be related to the utilization of plasma thiols (Figure 2B) as a longterm peripheral antioxidant control against possible oxidative damage. Thus, the interesting aspects of the results illustrated in Figure $2 \mathrm{~A}$ and $\mathrm{B}$ are the ability of plasma to recover from oxidative damage and the regeneration of thiols after 30-day reperfusion, even after reduction of about $50 \%$ of thiol levels after 5-day reperfusion.

The effect of $60 \mathrm{~min}, 1$ and 2 days of reperfusion on plasma of rats submitted to a 10 -min brain ischemic episode after preconditioning $\left(2^{\prime}+10^{\prime}\right)$ is demonstrated in Figure $3 \mathrm{~A}$ and $\mathrm{B}$. As shown in Figure 3A, when chemiluminescence emission was measured, plasma of rats submitted to 10-min injurious ischemia after a preconditioning time $\left(2^{\prime}+1-\right.$ day reperfusion) was not significantly different from controls or from the precondi- tioned group. Reperfusion after preconditioning to brain ischemia did not change plasma chemiluminescence emission (Figure $3 \mathrm{~A}$ ). Plasma thiol content was measured under the same conditions of reperfusion after preconditioning to brain ischemia used to measure plasma chemiluminescence emission. Figure 3B shows that preconditioning $\left(2^{\prime}+1\right.$-day reperfusion) significantly decreased plasma SH groups when compared to control. On the other hand, 10-min ischemia after preconditioning $\left(2^{\prime}+10^{\prime}\right)$ was able to maintain plasma $\mathrm{SH}$ groups at control

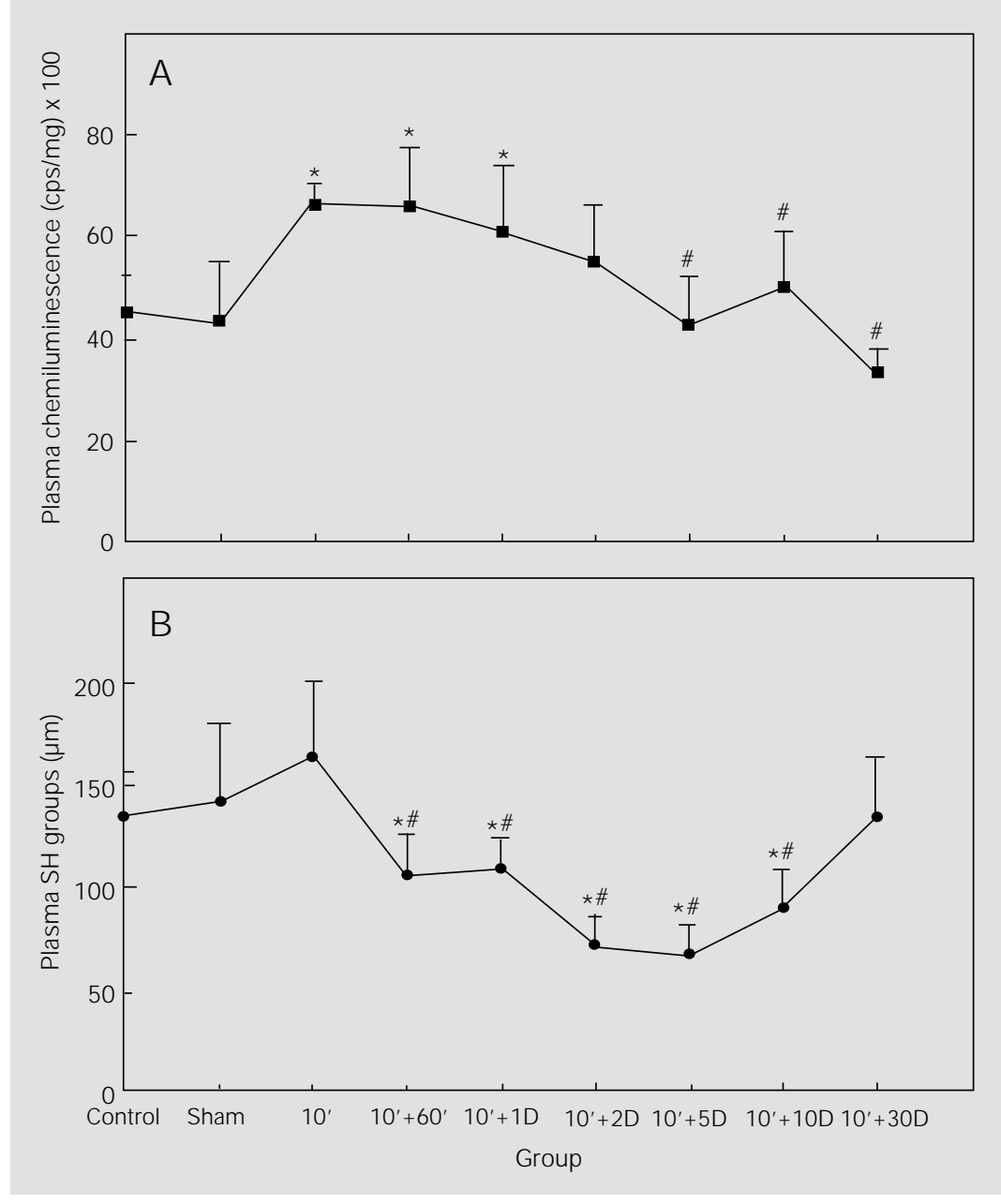

Figure 2 - Effect of 10-min (10') transient forebrain ischemia and reperfusion in rats on tertbutyl hydroperoxide-initiated chemiluminescence of plasma (A) and plasma thiols (B). Rats were submitted to 10-min (10') ischemia followed by 60 min or 1, 2, 5, 10 and 30 days (D) of reperfusion. Data represent the mean $\pm \mathrm{SD}$ for six to eight different animals. $* \mathrm{P}<0.05$ compared to control groups. $\mathrm{P}_{\mathrm{P}}<0.05$ compared to the 10-min (10') ischemic group (Duncan multiple range test). SH, Sulfhydryl. 
levels. Since 2-min ischemia followed by 1 day reperfusion induces tolerance to a longer and injurious ischemic episode (10 $\mathrm{min})$, we suggest that in this situation plasma SH groups were decreased as sacrificial antioxidants (14), probably to protect more important targets such as DNA and lipids against oxidative damage. Thus, these thiols are damaged but there are so many in plasma that the effect might be biologically nonsignificant (14). Probably, the plasma of rats submitted to 2 min of ischemia followed by 1 day of
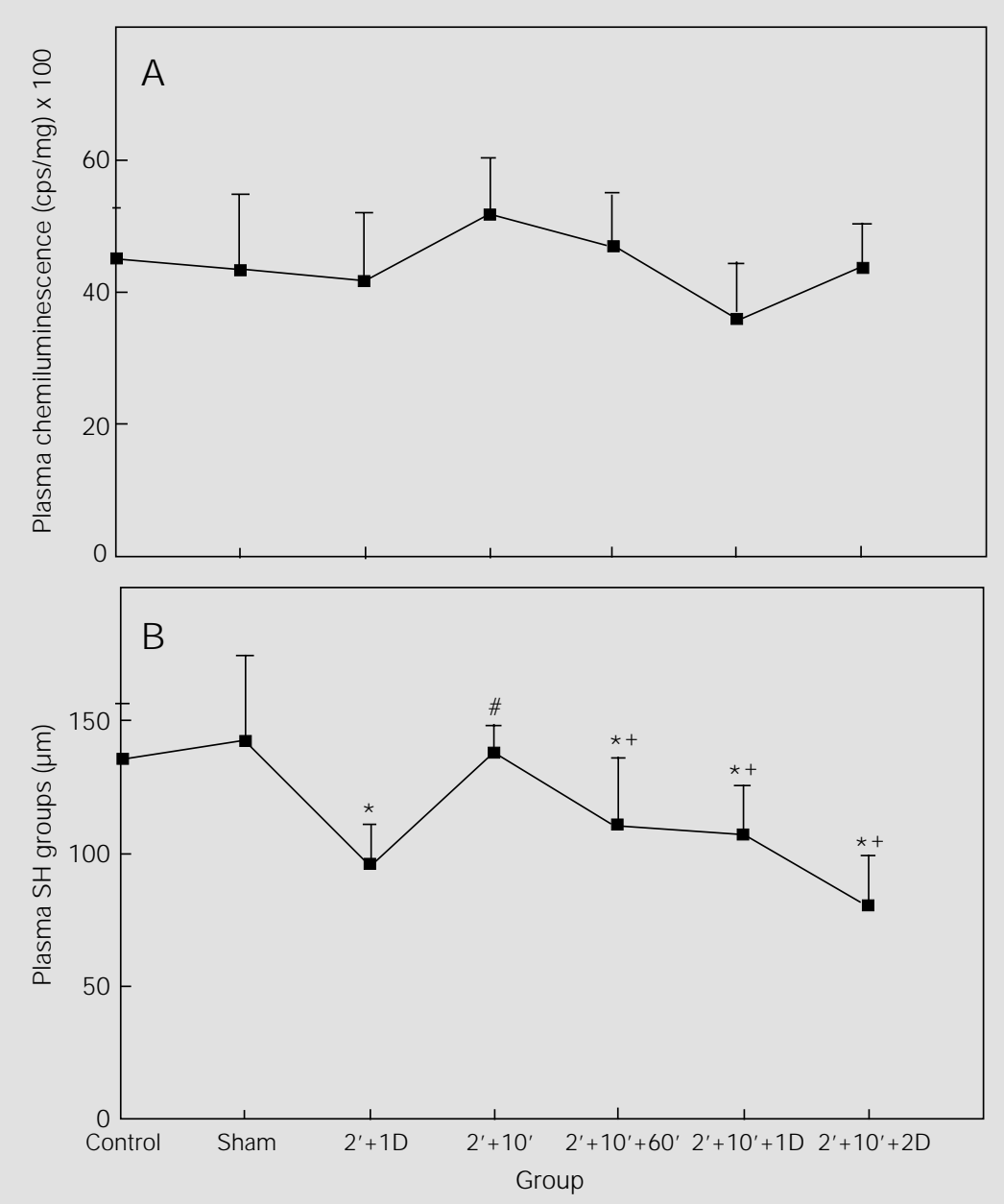

Figure 3 - Effect of brain ischemic preconditioning and reperfusion in rats on tert-butyl hydroperoxide-initiated chemiluminescence of plasma (A) and plasma thiols (B). Rats were submitted to 2-min (2') ischemia followed by 1-day (D) reperfusion ( $\left.2^{\prime}+1 D\right)$ and 10-min

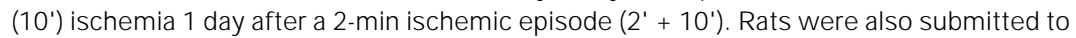
60 min or 1 and 2 days of reperfusion after the double-ischemic episode $\left(2^{\prime}+10^{\prime}\right)$. Data represent the mean $\pm \mathrm{SD}$ for six to nine different animals. ${ }^{*} \mathrm{P}<0.05$ compared to the control group. ${ }^{\#}<0.05$ compared to the $\left(2^{\prime}+1 D\right)$ group. ${ }^{+} \mathrm{P}<0.05$ compared to the $\left(2^{\prime}+10^{\prime}\right)$ group (Duncan multiple range test). SH, Sulfhydryl. reperfusion and to the double-ischemic episode did not show an increase in chemiluminescence emission because plasma SH groups were consumed in the first situation. Nevertheless, the results for plasma of rats submitted to 60 min or 1 and 2 days of reperfusion after preconditioning to brain ischemia $\left(2^{\prime}+\right.$ 10 ') indicate that in this situation reperfusion causes a loss of SH groups (Figure 3B).

By comparing Figure $3 \mathrm{~A}$ and $\mathrm{B}$ with others, we suggest that the permanence of plasma chemiluminescence emission at control levels may be associated with the consumption of plasma thiols. Interestingly, it is clear that plasma was protected from oxidative damage by a longer ischemic episode (10 min) and reperfusion when brain ischemic preconditioning occurred and, probably, when thiols were decreased (Figure 3A and $\mathrm{B})$. The results illustrated in Figure $3 \mathrm{~A}$ can be compared to the results in Figure 2A that shows a significant increase of plasma chemiluminescence emission when rats were submitted to 10-min ischemia and reperfusion without the protection of the preconditioning time.

The findings of our study are that brain ischemia and reperfusion produce peripheral alterations demonstrated in plasma that are probably related to an increased formation of reactive oxygen species. Furthermore, it seems that brain ischemic preconditioning, as a phenomenon responsible for the protection of the central nervous system against injurious ischemic episodes and reperfusion (21), also reduced plasma oxidative damage as assessed by chemiluminescence emission. In any case, the determination of plasma thiols may indicate a relationship between the consumption of SH groups and the permanence of plasma chemiluminescence emission at control levels. This relationship could be seen when rats were submitted to reperfusion after 2-min (Figure $1 \mathrm{~A}$ and $\mathrm{B}$ ) or 10-min ischemia (Figure $2 \mathrm{~A}$ and $\mathrm{B}$ ) and mainly after the preconditioning time followed by 10-min ischemia and re- 
perfusion (Figure $3 \mathrm{~A}$ and $\mathrm{B}$ ), which suggests that reperfusion is related to the reduction of thiols and, probably, to normalization of plasma chemiluminescence emission as much after single ischemic episodes as after doubleischemic episodes (preconditioning). Thus, the results may indicate that peripheral oxidative damage is associated with brain ischemia and reperfusion and that ischemic preconditioning induces protective effects. Considering the literature and our results, it seems that brain ischemia and preconditioning cause injurious and protective effects, respectively, both in the central nervous system and in the bloodstream, that could be a possible peripheral indicator of central events.

It has been suggested that neuronal damage in brain ischemia and reperfusion is partly due to oxidative damage caused by free radical formation (22). Free radicals are extremely reactive compounds that can react with lipids, enzymes $(23,24)$ or DNA to produce various harmful effects (25). Recent studies have shown the simultaneous production of $\mathrm{NO}$ and superoxide anion $\left(\mathrm{O}_{2}{ }^{\bullet-}\right)$ within reperfused ischemic cerebral tissue that might lead to the formation of cytotoxic peroxynitrite or an oxidant derived from it (26). Neuronal produced peroxynitrite may diffuse to the bloodstream (26), where it induces oxidative damage by its effects in decreasing the levels of antioxi- dants such as thiol groups $(27,28)$. For this reason, thiols have been regarded as sacrificial antioxidants in plasma (14), playing a role in the decreased mortality from coronary heart disease (29). We suggest that after brain ischemia, ischemic preconditioning and reperfusion, the reduction of thiols is probably related to a protection against plasma oxidative damage. Thus, the reduction of thiols in plasma is related to their antioxidative capacity, since oxidative damage revealed by chemiluminescence emission has been considered to be a consequence of reactive oxygen species generation (18). Strubelt and colleagues (30) demonstrated that thiols are related to protection against liver ischemia and hypoxia-reoxygenationinduced oxidative damage.

Since neuronal death is greatly reduced when ischemic preconditioning precedes an injurious ischemic episode and reperfusion (21), we suggest that sacrifice reactions of plasma SH groups with reactive oxygen species lead to oxidative reduction of thiols, an event implying the reduction of plasma oxidative damage by preconditioning. In conclusion, although much work is needed to determine which oxidants participate in the development of plasma injury during brain ischemia, the present results confirm the hypothesis that preconditioning reduces peripheral oxidative damage associated with brain ischemia and reperfusion.

\section{References}

1. Watanabe T, Yuki S, Egawa M \& Nishi H (1994). Protective effects of $\mathrm{MCl}-186$ on cerebral ischemia: possible involvement of free radical scavenging and antioxidant actions. J ournal of Pharmacology and Experimental Therapeutics, 268: 1597-1604.

2. Kinouchi H, Epstein CJ , Mizui T, Carlson E, Chen SF \& Chan PH (1991). Attenuation of focal cerebral ischemic injury in transgenic mice overexpressing CuZn superoxide dismutase. Proceedings of the National Academy of Sciences, USA, 88:
11158-11162.

3. Globus MYT, Busto R, Lin B, Schnippering $H \&$ Ginsberg MD (1995). Detection of free radical activity during transient global ischemia and recirculation: effects of intraischemic brain temperature modulation. J ournal of Neurochemistry, 65: 12501256.

4. Traystman RJ, Kirsch J R \& Koehler RC (1991). Oxygen radical mechanism of brain injury following ischemia and reperfusion. J ournal of Applied Physiology, 71
(Suppl): 1185-1195.

5. Yang C, Lin N, Tsai $P$, Liu L \& Kuo J (1996). In vivo evidence of hydroxyl radical formation induced by elevation of extracellular glutamate after cerebral ischemia in the cortex of anesthetized rats. Free Radical Biology and Medicine, 20: 245-250.

6. Legrand C, Woimant F \& Caen J (1991). Platelet surface glycoprotein changes in patients with cerebral ischemia. J ournal of Experimental and Clinical Hematology, 
33: 497-500.

7. Isaka Y, Kimura K, Uehara A, Hashikawa K, Mieno M, Matsumoto M, Handa N, Nakabayashi S, Imaizumi M \& Kamada T (1989). Platelet aggregability and in vivo platelet deposition in patients with ischemic cerebrovascular disease. Evaluation by indium-111-platelet scintigraphy. Thrombosis Research, 56: 739-749.

8. Tanahashi N, Tomita M, Kobari M, Takeda H, Yokoyama M, Takao M \& Fukuuchi Y (1996). Platelet activation and erythrocyte aggregation rate in patients with cerebral infarction. Clinical Hemorheology, 16: 497-505.

9. Kumura E, Kosaka H, Shiga T, Yoshimine $T$ \& Hayakawa T (1994). Elevation of plasma nitric oxide end products during focal cerebral ischemia and reperfusion in the rat. J ournal of Cerebral Blood Flow and Metabolism, 14: 487-491.

10. Salvemini D \& Botting R (1993). Modulation of platelet function by free radicals and free-radical scavengers. Trends in Pharmacological Sciences, 14: 36-42.

11. Sneddon J M \& Vane J R (1988). Endothelium-derived relaxing factor reduces platelet adhesion to bovine endothelial cells. Proceedings of the National Academy of Sciences, USA, 85: 2800-2804.

12. Losano G, Gattullo D \& Pagliaro P (1996). Myocardial, neural and vascular aspects of ischemic preconditioning. Life Sciences, 59: 1185-1192.

13. Llesuy S, Milei J, Gonzalez Flecha B \& Boveris A (1990). Myocardial damage induced by doxorubicins: hydroperoxide-initiated chemiluminescence and morphology. J ournal of Free Radicals in Biology and Medicine, 8: 259-264.

14. Halliwell B (1988). Albumin - an important extracellular antioxidant? Biochemical Phar- macology, 37: 569-571.

15. Das DK \& Maulik N (1994). Antioxidant effectiveness in ischemia-reperfusion tissue injury. Methods in Enzymology, 233: 601-610.

16. Pulsinelli WA, Brierley MD \& Plum $F$ (1982). Temporal profile of neuronal damage in a model of transient forebrain ischemia. Annals of Neurology, 1: 491-498.

17. Netto CA, Hodges $H$, Sinden J D, Le Peillet E, Kershaw T, Sowinski P, Meldrum BS \& Gray J A (1993). Effects of fetal hippocampal field grafts on ischaemic-induced deficits in spatial navigation in the water maze. Neuroscience, 54: 69-92.

18. Flecha BG, Llesuy S \& Boveris A (1991). Hydroperoxide-initiated chemiluminescence: an assay for oxidative stress in biopsies of heart, liver, and muscle. Free Radical Biology and Medicine, 10: 93-100.

19. Hu M (1994). Measurement of protein thiol groups and glutathione in plasma. Methods in Enzymology, 233: 380-385.

20. Bradford MM (1976). A rapid and sensitive method for the quantification of microgram quantities of protein utilizing the principle of protein dye binding. Analytical Biochemistry, 72: 248-254.

21. Kitigawa $K$, Matsumoto $M$, Tagaya $M$, Hata R, Ueda $H$, Niinobe $M$, Handa $N$, Fukunaga R, Kimura K, Mikoshiba K \& Kamada T (1990). Ischemic tolerance phenomenon found in the brain. Brain Research, 528: 21-24.

22. Halliwell B (1992). Reactive oxygen species and the central nervous system. J ournal of Neurochemistry, 59: 1609-1623.

23. Vietta M, Frassetto SS, Battastini AMO, Klein AB, Moreira C, Dias RD \& Sarkis J J F (1996). Sensitivity of ATPase-ADPase activities from synaptic plasma membranes of rat forebrain to lipid peroxidation in vi- tro and the protective effect of vitamin $\mathrm{E}$. Neurochemical Research, 21: 299-304.

24. Frassetto SS, Dias RD \& Sarkis J J F (1997) Free radical-induced inhibition of ATP diphosphohydrolase activity (EC 3.6.1.5) from rat blood platelets. Biochemistry and Molecular Biology International, 41: 161168.

25. Siesjo BK, Agardh C-D \& Bengtsson F (1989). Free radicals and brain damage. Cerebrovascular and Brain Metabolism Reviews, 1: 165-211.

26. Kumura E, Yoshimine T, Iwatsuki $K$, Yamanaka K, Tanaka S, Hayakawa T, Shiga T \& Kosaka H (1996). Generation of nitric oxide and superoxide during reperfusion after focal cerebral ischemia in rats. American J ournal of Physiology, 270 (Cell Physiology, 39): C748-C752.

27. Van $\operatorname{der}$ Vliet A, Smith D, O'Neill CA, Kaur $\mathrm{H}$, Darley-Usmar V, Cross CE \& Halliwell $B$ (1994). Interactions of peroxynitrite with human plasma and its constituents: oxidative damage and antioxidant depletion. Biochemical J ournal, 303: 295-301.

28. Vásquez-Vivar J, Santos AM, J unqueira VBC \& Augusto O (1996). Peroxynitritemediated formation of free radicals in human plasma: EPR detection of ascorbyl, albumin-thiol and uric acid-derived free radicals. Biochemical J ournal, 314: 869876.

29. Luoma PV, Nayha S, Sikkila K \& Hassi J (1995). High serum alpha-tocopherol, albumin, selenium and cholesterol, and low mortality from coronary heart disease in northern Finland. J ournal of Internal Medicine, 237: 49-54.

30. Strubelt O, Younes M \& Li Y (1994). Protection by albumin against ischaemia- and hypoxia-induced hepatic injury. Pharmacology and Toxicology, 75: 280-284. 